\title{
Hashimoto's Encephalopathy in A 30 Years Old Healthy Male: Case Report and Literature Review
}

\author{
Jnadi Mohammed Madkhali' ${ }^{*}$, Ammar Abdullah Hakami', Salha Marzoog Alharbi
}

${ }^{1}$ Medical Intern, Jazan University, Jazan, Saudi Arabia. ${ }^{2}$ Neurology Consultant, Department of Internal Medicine, King Fahd General Hospital, Jeddah, Saudi Arabia.

*Corresponding author: Jnadi Mohammed Madkhali, Mobile phone: +966534423567, Email: JNADY888@GMAIL.COM

\begin{abstract} memory impairment, depressed mood and psychosis. the acute confusional state. after initiation of intravenous Methylprednisolone therapy.

\section{INTRODUCTION}

Hashimoto's thyroiditis (HT) is an autoirnmune disorder in which the thyroid gland gradually destroyed by the immune system ${ }^{(1)}$. It is the most common cause of hypothyroidism worldwide, with serious consequences if unrecognized and untreated (2). While rare, autoimmune thyroiditis may be associated with metabolic encephalopathy ${ }^{(3)}$. A rare form of neuroendocrine disorder associated with autoimmune thyroiditis is called Hashimoto Encephalopathy (HE) ${ }^{(4)}$. It is consisting of various neuropsychological symptoms including seizure, confusion, personality changes, together with high levels of serum thyroid-related autoantibodies ${ }^{(5)}$. Its diagnosis based mainly on exclusion. However, the exact mechanism, presentation and management of patients with (HE) still not fully understood. The present case aims to shed light on this gap.
\end{abstract}

Context: Hashimoto's encephalopathy is a rare neurological disorder associated with autoimmune thyroiditis. It occurs primarily in the fifth decade of life. However, different age groups may also develop the disease, with serious consequences if unrecognized and untreated. Clinically, it comprises two forms: a sudden vasculitic, and progressive subacute type with wide neuropsychiatric manifestations including confusion, seizures, short term

Objective: The objective of this report is to present an unusual case of Hashimoto's encephalopathy presented with

Case presentation: We report the case of a 30-year-old male medically free presented with acute behavioral changes and confusion. Serological studies revealed elevated thyroid peroxidase antibodies levels. In addition, EEG findings of slow background. He was diagnosed as a case of Hashimoto's encephalopathy and he was improved

Conclusion: Hashimoto's encephalopathy can present with a challenging clinical picture that may go unrecognized for a long time. Therefore, one should keep in mind the importance of evaluating thyroid function and thyroid antibodies levels in patients presented with unexplained neurocognitive and psychiatric manifestations.

Keywords: Autoimmune thyroiditis, Hashimoto's encephalopathy, Intravenous Steroid, Case report.

\section{Case presentation}

The consent was taken from the patient as well as from the Hospital Research Committee. This is a 30-year-old male who isn't known to have any illness before, presented to the emergency department of King Fahd General Hospital, Jeddah, Saudi Arabia, with his relative complaining of behavioral changes for 1 week, associated with headache, low mood, disturbed sleep, nausea and decrease oral intake. The condition started 10 days back when he had an intermittent low-grade fever that lasted for 2 days then spontaneously resolved. Then the patient developed acute behavioral changes, his family noticed that he is crying, not talking or communicating. On the day of admission, his condition worsened and he started to become more aggressive for which his family brought him to the hospital. According to relatives, the patient had no history of convulsion, weakness, decreased level of consciousness or trauma. The patient had no other symptoms. No history of smoking, alcohol abuse or drug addiction. There was no past medical history of a similar attack. His family history had no similar conditions or chronic illnesses, or contact with ill people. The patient also had no history of a long term or recent medications. He had no history of allergy to known food or medications. At presentation, the patient was confused, had delayed emotional response, depressed mood, poor eye contact and no verbal communication but vitally stable. The mental state was difficult to be assessed as the patient was aggressive and agitated. Neurological examination revealed intact cranial nerves, sensory and motor functions. He had no abnormal movements or twitches as well as no signs of hypothyroidism. A blood test taken for laboratory workup (Table 1).

The laboratory results showed high WBC (13x10/L), high CK (1283 U/L), low potassium (3.3 $\mathrm{mmol} / \mathrm{l})$, otherwise, it was unremarkable. The initial plain CT brain showed no evidence of acute brain insult. Then LP requested, initially patient was not cooperative, 2 days later LP performed and CSF was taken for analysis and culture (Table 2). The results revealed high total protein, normal glucose, and zero 
WBC count. Moreover, urine toxicology screen and serology requested and both were unremarkable (Table 3).

Later on, further workup including EEG, thyroid function test and thyroid antibodies were requested. EEG revealed a diffuse slow background. Thyroid functions showed high TSH $(7.7 \mathrm{IU} / \mathrm{ml})$, normal T3 $(2.5 \mathrm{pg} / \mathrm{ml})$ and $\mathrm{T} 4(1.2 \mathrm{ng} / \mathrm{dl})$. In addition, thyroid peroxidase antibodies were highly abnormal (155.75
IU/ml) (Table 4). Therefore, supported by the clinical presentation, thyroid studies, imaging and EEG, the diagnosis of Hashimoto's encephalopathy was made. Accordingly, the patient started on Methylprednisolone 1g IV once daily for 5 days. Later on, marked improvement noticed in his condition during the short term follow up after initiation of steroid therapy.

\begin{tabular}{|c|c|}
\hline Laboratory data & Result \\
\hline \multicolumn{2}{|l|}{$\mathrm{CBC}$} \\
\hline RBC & $4.7 \times 10^{12} / \mathrm{L}$ \\
\hline WBC & $13 \times 10^{9} / \mathrm{L}$ \\
\hline Hemoglobin & $13.4 \mathrm{~g} / \mathrm{dl}$ \\
\hline $\mathrm{MCV}$ & $86 \mathrm{fL}$ \\
\hline Platelet & $381 \times 10^{9} / \mathrm{L}$ \\
\hline \multicolumn{2}{|l|}{ Liver function test } \\
\hline AST (SGOT) & $64 \mathrm{U} / \mathrm{L}$ \\
\hline ALT (SGPT) & $19 \mathrm{U} / \mathrm{L}$ \\
\hline Total bilirubin & $0.29 \mathrm{mg} / \mathrm{dl}$ \\
\hline Direct bilirubin & $0.1 \mathrm{mg} / \mathrm{dl}$ \\
\hline \multicolumn{2}{|l|}{ Renal function test } \\
\hline Creatinine & $0.97 \mathrm{mg} / \mathrm{dl}$ \\
\hline BUN & $14 \mathrm{mg} / \mathrm{dl}$ \\
\hline \multicolumn{2}{|l|}{ Cardiac profile } \\
\hline CK & $1283 \mathrm{U} / \mathrm{L}$ \\
\hline CK-MB & $5.3 \mathrm{ng} / \mathrm{ml}$ \\
\hline \multicolumn{2}{|l|}{ Electrolytes } \\
\hline $\mathrm{Na}$ & $137 \mathrm{mmol} / \mathrm{L}$ \\
\hline K & $3.3 \mathrm{mmol} / \mathrm{L}$ \\
\hline \multicolumn{2}{|l|}{ Coagulation profile } \\
\hline PTT without anticoagulant & 39.93 seconds \\
\hline PT without anticoagulant & 11.83 seconds \\
\hline INR without anticoagulant & 1.02 seconds \\
\hline
\end{tabular}

\begin{tabular}{|l|l|}
\hline \multicolumn{2}{|l|}{ Table 2: Cerebrospinal fluid results } \\
\hline Culture \& Susceptibility test & No growth \\
\hline Glucose & $61 \mathrm{mg} / \mathrm{dl}$ \\
\hline Total protein & $48.5 \mathrm{mg} / \mathrm{dl}$ \\
\hline Color & Colorless \\
\hline Turbidity & Clear \\
\hline WBC count & 0 Cells/UL \\
\hline
\end{tabular}

Table 3: Serology workup

Viral markers

Hepatitis B surface antigen

Hepatitis C surface antibody

HIV workup

HIV antibody

Negative

Negative

Negative 


\begin{tabular}{|l|l|}
\hline \multicolumn{2}{|l|}{ Table 4: Thyroid workup } \\
\hline Thyroid function test & $7.7 \mathrm{IU} / \mathrm{ml}$ \\
\hline TSH & $2.5 \mathrm{pg} / \mathrm{ml}$ \\
\hline T3 & $1.2 \mathrm{ng} / \mathrm{dl}$ \\
\hline T4 & $155.75 \mathrm{IU} / \mathrm{ml}$ \\
\hline Thyroid autoantibodies &
\end{tabular}

\section{DISCUSSION}

Hashimoto encephalopathy is a rare syndrome of neurological and neuropsychiatric deficits associated with high levels of thyroid autoantibodies ${ }^{(5)}$. Only 85 cases reported in the literature in a systematic review published in $2003^{(6)}$. The condition occurs more commonly between the age of 44 to 46 years ${ }^{(7)}$. However, it can affect any age group including children ${ }^{(8)}$, adults ${ }^{(9)}$ and elderly $(10,11)$ with serious consequences if unrecognized and untreated ${ }^{(2)}$. In the present case, we are reporting a case of $\mathrm{HE}$ in a 30-year-old male patient. The pathogenesis of HE does not appear to be related to the thyroid gland status, which varies in patients with HE. Two recent studies revealed around $30 \%$ of patients had subclinical hypothyroidism, $20 \%$ had hypothyroidism, less than $8 \%$ had hyperthyroidism and almost $40 \%$ were euthyroid ${ }^{(6,7)}$. Development of neuropsychiatric features may precede the onset of Hashimoto's thyroiditis ${ }^{(12)}$. There is no evidence to explain the effect of thyroid antibodies on the brain tissues. In addition, there is no correlation between levels of these antibodies and severity of neurological symptoms ${ }^{(13)}$. However, the presence of elevated thyroid peroxidase antibodies levels remains important to diagnose $\mathrm{HE}$ and indicates thyroid autoimmunity ${ }^{(6,7)}$. Although the disease has a heterogeneous presentation, 2 clinical forms have been described. The vasculitic type, which is characterized by seizures and focal neurological deficits and the diffuse progressive type which has marked behavioral changes, short term memory impairment, confusion, somnolence, depressed mood and psychosis ${ }^{(14)}$. However, neither presentation is exclusive and overlap may occur over the course of the disease. Brain et al. ${ }^{(4)}$ reported the first case of neuropsychiatric manifestations associated with autoimmune thyroiditis in 1966 . He described a 40year-old patient known case of Hashimoto's thyroiditis who subsequently developed neurological deficits and abnormal behavior. Further, Shaw et al. (11) reported 5 cases with subacute encephalopathy and elevated thyroid auto antibodies and the term "Hashimoto Encephalopathy" was proposed. In addition, Berger et al. ${ }^{(8)}$ reported an 11-year-old boy who had history of refractory epilepsy followed by a progressive decline in his cognitive function, manifested by a significant decline in school performance, aggression and hallucination. The patient experienced extensive evaluation until $\mathrm{HE}$ was diagnosed. In this case report, our patient's clinical manifestations are more consistent with the diffuse progressive form of presentation, which is more common. Diagnosis of HE mainly depends on excluding all possible differential diagnoses of acute to sub-acute neuropsychiatric manifestations, including traumatic, infectious, drug induced or autoimmune causes. CSF analysis in Hashimoto's encephalopathy usually shows high protein, normal glucose and zero WBC count in more than $75 \%$ of cases ${ }^{(10)}$. This was consistent with CSF results in our patient (Table 2). Furthermore, EEG changes in HE were nonspecific. It may show slow background activity in more than $90 \%$ of the patients ${ }^{(15)}$. After excluding all the possible etiologies, together with high serum thyroid peroxidase antibodies and EEG evidence of a slow background, the diagnosis of HE was established in the present case. Steroid therapy was proved to be the most effective treatment method for $\mathrm{HE}$, and even some authors suggested to change HE definition to "corticosteroid-responsive encephalopathy associated with Hashimoto's thyroiditis" (6,11,16). Accordingly, our patient started on Methylprednisolone with marked improvement in his condition during the short term follow up.

\section{CONCLUSION}

Hashimoto's encephalopathy frequently presents with a diverse clinical picture, including seizures, focal neurological deficits, confusion, depression, delusions and hallucinations. Such condition may go unrecognized for a long time. Therefore, one should keep in mind the importance of evaluating thyroid function and thyroid antibodies levels in patients presented with unexplained neurocognitive and psychiatric manifestations.

\section{Consent}

Written informed consent from the patient was obtained for publication.

\section{Data availability statement}

The data used in this study are available from the corresponding author upon request.

\section{Conflict of interest}

The authors declare they have no conflicts of interest. 


\section{REFERENCES}

1. Del Prete G, Vercelli D, Tiri A, Maggi E, Mariotti S, Pinchera A, Ricci M, Romagnani S (1986): In vivo activated cytotoxic $\mathrm{T}$ cells in the thyroid infiltrate of patients with Hashimoto's thyroiditis. Clin Exp Immunol., 65:140-7.

2. Fiore E, Rago T, Latrofa F, Provenzale M, Piaggi P, Delitala A, Scutari M (2011): Hashimoto's thyroiditis is associated with papillary thyroid carcinoma: role of TSH and of treatment with Lthyroxine. Endocr Relat Cancer., 18(4):429-437.

3. Richard C, Michael K, Richard D et al. (1982): Psychiatric manifestations of Hashimoto's thyroiditis. Psychosomatics., 23(4):337-342.

4. Lord B, Keith B (1966): Hashimoto's disease and encephalopathy. Lancet (London, England), 288(7462):512-514.

5. Tamagno G, Federspil G, Murialdo G (2006): Clinical and diagnostic aspects of encephalopathy associated with autoimmune thyroid disease (or Hashimoto's encephalopathy). Intern Emerg Med., 1(1):15-23.

6. Chong J, Rowland L, Utiger R (2003): Hashimoto encephalopathy: syndrome or myth? Arch Neurol., 60(2):164-171.

7. Ferracci F, Carnevale A (2006): The neurological disorder associated with thyroid autoimmunity. J Neurol., 253(8):975-984.

8. Berger I, Castiel Y, Dor $T$ (2010): Paediatric Hashimoto encephalopathy, refractory epilepsy and immunoglobulin treatment - unusual case report and review of the literature. Acta Pædiatrica, 99(12):19035.

9. Kothbauer-Margreiter I, Sturzenegger M, Komor
J, Baumgartner R, Hess C (1996): Encephalopathy associated with Hashimoto thyroiditis : diagnosis and treatment. J Neurol., 243(8):585-93.

10. Canelo-aybar C, Loja-oropeza D, Cuadraurteaga J, Romani-romani F (2010): Hashimoto's encephalopathy presenting with neurocognitive symptoms: a case report. J Med Case Rep., 4:337.

11. Shaw $P$, Walls $T$, Newman $P$, Cleland $P$, Cartlidge N (1991): Hashimoto's encephalopathy: a steroidresponsive disorder associated with high anti-thyroid antibody titers-report of 5 cases. Neurology, 41(2):228-33.

12. Peschen-Rosin R, Schabet M, Dichgans J (1999): Manifestation of Hashimoto's encephalopathy years before the onset of thyroid disease. Eur Neurol., 41(2):79-84.

13. Ferracci F, Bertiato G, Moretto $G$ (2004): Hashimoto's encephalopathy: epidemiologic data and pathogenetic considerations. J Neurol Sci., 217(2):165-168.

14. Leigh H, Kramer S (1984): The psychiatric manifestations of endocrine disorders. Adv Intern Med., 29:413-445.

15. Rodriguez A, Jicha G, Steeves T et al. (2006): EEG changes in a patient with steroid-responsive encephalopathy associated with antibodies to thyroperoxidase (SREAT, Hashimoto's encephalopathy). J Clin Neurophysiol., 23(4):371373.

16. Mahmud F, Lteif A, Renaud D, Reed A, Brands C (2003): Steroid-responsive encephalopathy associated with Hashimoto's thyroiditis in an adolescent with chronic hallucinations and depression: case report and review. Pediatrics, 112(3):686-90. 